
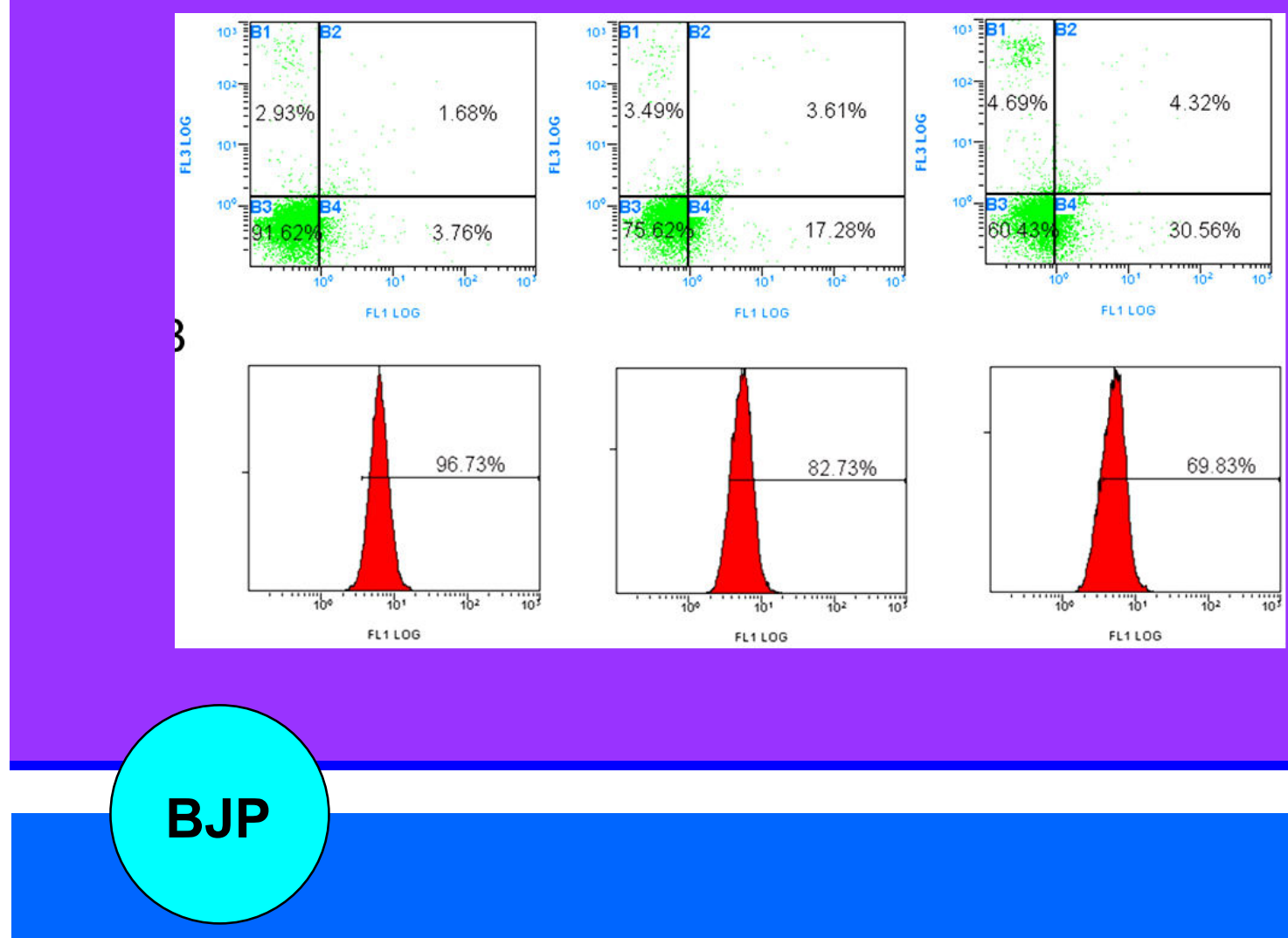

Bangladesh Journal of Pharmacology

Research Article

Tubeimoside- 1 inhibits growth via the induction of cell cycle arrest and apoptosis in human melanoma A375 cells 
Abstracted/indexed in Academic Search Complete, Asia Journals Online, Bangladesh Journals Online, Biological Abstracts, BIOSIS Previews, CAB Abstracts, Current Abstracts, Directory of Open Access Journals, EMBASE/Excerpta Medica, Google Scholar, HINARI (WHO), International Pharmaceutical Abstracts, Open J-gate, Science Citation Index Expanded, SCOPUS and Social Sciences Citation Index;

ISSN: $1991-0088$

\title{
Tubeimoside-1 inhibits growth via the induction of cell cycle arrest and apoptosis in human melanoma A375 cells
}

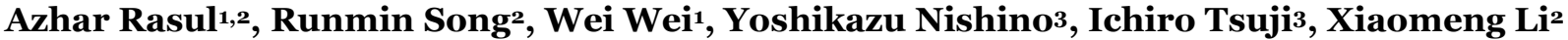 \\ and Jiang Li ${ }^{1}$ \\ ${ }^{1}$ Dental Hospital, Jilin University, Changchun 130041; ${ }^{2}$ The Key Laboratory of Molecular Epigenetics of MOE, \\ Institute of Genetics and Cytology, School of Life Sciences, Northeast Normal University, Changchun 130024, \\ China; ${ }^{3}$ Department of Public Health, Tohoku University, Sendai 9808576, Japan.
}

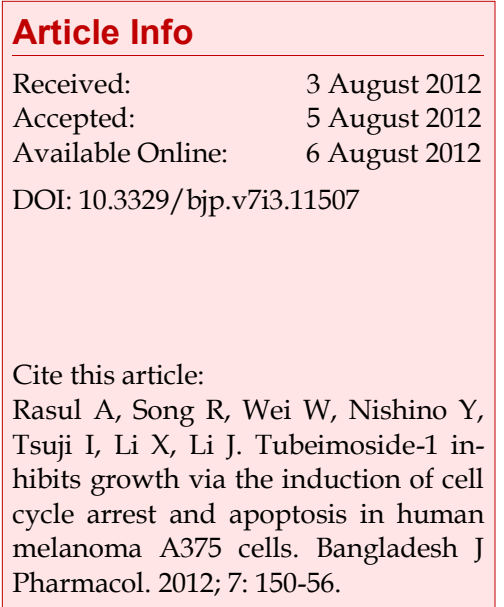

\begin{abstract}
Tubeimoside-1 (TBMS1) has been shown to possess promising anticancer potential against various cancer cells. However, its effect against melanoma cells remained enigmatic. In this study, for the first time, we investigated the effect of TBMS1 on cell proliferation, cell cycle, and apoptosis in melanoma A375 cells. In order to examine the effects of TBMS1 on cell proliferation, cell cycle, apoptosis, we performed MTT assays and flow cytometry analysis. The results revealed that TBMS1 decreased the cell viability of melanoma A375 cells. Flow cytometric analysis demonstrated that TBMS1 markedly induced apoptosis of A375 cells and arrested cell cycle at G2/M phase in a dosedependent manner. Induction of apoptosis involved a decrease in mitochondrial membrane potential, down-regulation of Bcl-2 and up- regulation of Bax before culminating in apoptosis in TBMS1-treated A375 cells. These in vitro results suggested that TBMS1 should be further examined for in vivo activity and molecular mechanism in human melanoma.
\end{abstract}

\section{Introduction}

In the past decades, natural products have become most important source of potential anticancer agents and several compounds, which were originally isolated from plants such as paclitaxel, camptothecin, vinca alkaloids, and etoposide have potential application in cancer chemotherapy, therefore, plants are considered as one of the most important sources for the development of novel anti-cancer drugs (Amin et al., 2009; Cragg and Newman, 2005). Triterpenoid saponins possess a variety of biological activities and considerable attention in pharmacological research due to their anti-fungal, antivirus, and anti-inflammatory activities (Magadula and Erasto, 2009; Woldemichael and Wink, 2001). Tubeimoside-1 (TBMS1), a triterpenoid saponin has been reported to have wide spectrum of biological effects, including anti-inflammatory, anti-tumorigenic and anti-HIV activities (Yu et al., 1994; Yu et al., 1992). Furthermore, TBMS1 has anti-proliferative effect and induced apoptosis in cancer cells such as lung cancer (Zhang et al., 2011), cervical cancer (Xu et al., 2009), nasopharyn-geal carcinoma (Ma et al., 2008; Weng et al., 2003) and leukemia HL-60 cells (Yu et al., 1996). However, the cytotoxic effects of TBMS1 on melanoma and its mechanism were still unknown.

Melanoma is an increasingly common and potentially lethal malignancy. Melanoma treatment is widely studied by single and combination of anticancer drugs to prevent the spread of metastasis (Gray-Schopfer et al., 2007). The chemotherapeutic treatment of melanoma is done by using several compounds such as single \{dacarbazine, response rates of 11-25\% and short survival time\} or in combinations \{dacarbazine, cisplatinum and vindesine, response rate of $40 \%$, including com- 


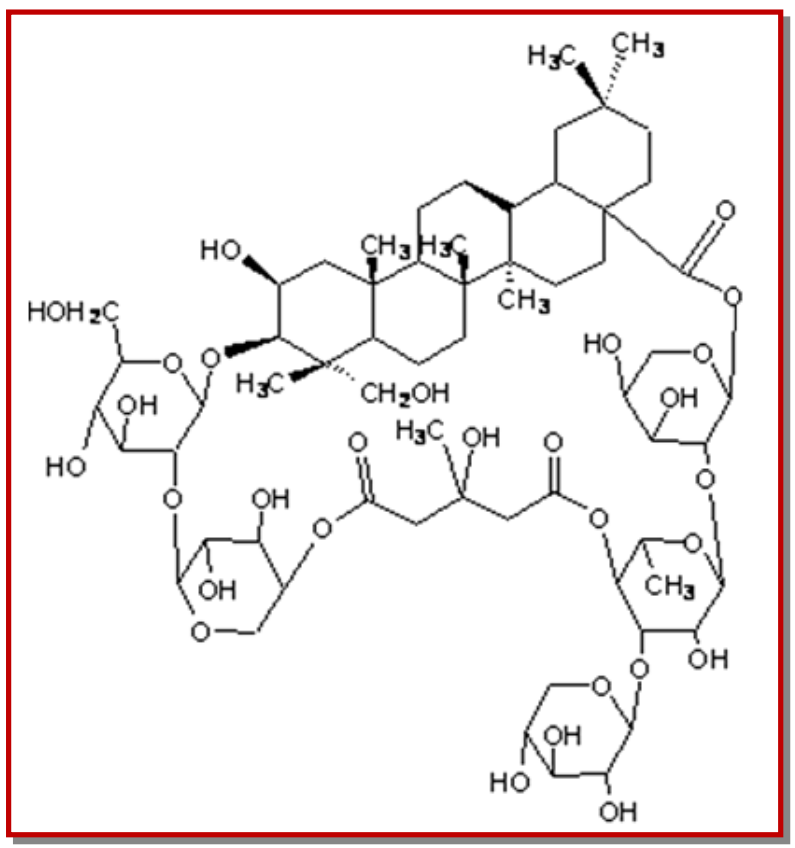

Figure 1: Structure of tubeimoside-1 (TBMS1)

plete response of $4 \%$ \} (Ives et al., 2007; Legha et al., 1996). These chemotherapeutic treatments were not potent enough against melanoma and development of novel compounds that can prevent metastasis is in progress (Hofmann et al., 2007). In the last few decades several natural compounds have shown a great promise in treatment of cancer and prevention of metastasis; most of the researchers are interested in and are focusing on finding a cure for cancer and several published reports have identified numerous dietary and botanical natural compounds that have chemopreventive potential (Rasul et al., 2012a). Therefore, novel therapeutic agents and treatment approaches are desired to improve the clinical outcome. For this purpose, natural compounds were screened against human melanoma A375 cells. TBMS1 was one of them which showed potent anti-proliferative effect against the malignant melanoma cells. Further characterization showed that TBMS1 effectively inhibited the proliferation of A375 cells through arresting cell cycle at G2/M phase and induction of apoptosis which is regulated by downregulation of Bcl-2 and up-regulation of Bax.

\section{Materials and Methods}

\section{Chemical and reagents}

Tubeimoside-1 (Figure 1) was purchased from the National Institute for the Control of Pharmaceutical and Biological Products (Beijing, China). Fetal bovine serum was purchased from Hangzhou Sijiqing Biological Engineering Materials Co., Ltd. DMEM, MTT [3'-(4,5dimethylthiazol-2-yl)-2,5-diphenyl tetrazolium bro- mide], propidium iodide (PI), Hoechst 33258, and dimethyl sulfoxide (DMSO) were purchased from Sigma Chemical Company (St. Louis, USA). Annexin VFITC Apoptosis Detection Kit was purchased from the Beyotime Institute of Biotechnology (Shanghai, China). Rabbit polyclonal anti-human Bcl-2, anti-and human Bax antibodies were purchased from Wuhan Boster Biological Technology Co., Ltd. Mouse anti- $\beta$-actin and anti-rabbit antibodies were purchased from Santa Cruz Biotechnology. Ponceou and cell lysis buffer for Western and IP were purchased from Bio SS Beijing. Rho123 was purchased from Eugene Co. (Oregon, U.S.A.).

\section{Cell culture}

Human melanoma A375 cells were propagated in DMEM nutrients mixture supplemented with 10\% FBS and antibiotics at $37^{\circ} \mathrm{C}$ in a humidified atmosphere with $5 \% \mathrm{CO}_{2}$ and $95 \%$ air. Cells were seeded in $10 \mathrm{~cm}$ culture dish and allowed to grow to approximately $70 \%$ confluence before experimentation.

\section{Cell proliferation assay}

The cytotoxic effects of the TBMS1 on the cells were determined by MTT assay as described previously (Rasul et al., 2011a; Rasul et al., 2011b). Briefly, A375 cells were seeded at a density of $1 \times 104$ cells per well in 96-well plates and were allowed to grow overnight. Cells were incubated with $100 \mu \mathrm{L}$ of complete culture medium containing 10, 20, 40, 60, 80, 100, and $200 \mu \mathrm{M}$ of TBMS1. After incubation for 24 hours, growth of cells was determined by adding $10 \mu \mathrm{L}$ MTT $(5 \mathrm{mg} / \mathrm{mL}$ in phosphate buffered saline) to each well and incubated for 4 hours. After removal of the medium, $150 \mu \mathrm{L}$ DMSO was added to each well and shaken carefully. The absorbance was read at a wavelength of $570 \mathrm{~nm}$ in a plate reader (ELX 800, BIO- TEK Instruments Inc.). The growth curve was plotted against mean values which were calculated using the following equation:

$\mathrm{I} \%=\left[\mathrm{A}_{570}\right.$ (control) $-\mathrm{A}_{570}$ (treated) $] / \mathrm{A}_{570}$ (control) *100

\section{Flow cytometric analysis of cell cycle}

For cell analysis, A375 cells were seeded in 12-well plates and then treated with 20 and $40 \mu \mathrm{M}$ of TBMS1 for 24 hours. After treatments, the percentages of cells in the different phases of cell cycle were evaluated by determining the DNA content after propidium iodide (PI) staining. Briefly, cells were washed with PBS, trypsinized and centrifuged at $1000 \mathrm{rpm}$ at $4^{\circ} \mathrm{C}$ for 5 min. Pellets were fixed overnight in $70 \%$ cold ethanol. After fixation, cells were washed twice with PBS and incubated in PBS containing RNase $(1 \mathrm{mg} / \mathrm{mL})$ for 10 min at room temperature. Finally, samples were stained with propidium iodide $(1 \mathrm{mg} / \mathrm{mL})$ for $30 \mathrm{~min}$ at $4^{\circ} \mathrm{C}$. Data acquisition was done by flow cytometry (EPICSXL - MCL, Beckman Coulter, US) using Cell Quest software. 


\section{Flow cytometric determination of apoptosis}

The apoptotic rate of A375 cells was examined by flow cytometry using annexin V-FITC/PI staining. Briefly, A375 cells were cultured in 6-well plates and allowed to attach overnight. Cells were treated with 20 and $40 \mu \mathrm{M}$ of TBMS1 for 24 hours. Then cells were collected, washed and resuspended in PBS. Apoptotic cell death was measured by double staining annexin V-FITC and PI using the Annexin V-FITC apoptosis detection kit (Beyotime Biotechnology Shanghai, China) according to the manufacturer's instructions. Flow cytometric analysis was performed immediately after staining. Data acquisition and analysis were performed by flow cytometry using Cell Quest software.

\section{Flow cytometric determination of mitochondrial mem- brane potential $(\Delta \Psi \mathrm{m})$}

To probe the changes in $\Delta \Psi \mathrm{m}, \mathrm{A} 375$ cells were stained with rhodamine $123(1 \mu \mathrm{M})$ after treatment of 20 and 40 $\mu \mathrm{M}$ of TBMS1 for 24 hours with control group. The fluorescence of rhodamine 123 was measured by flow cytometry with excitation and emission wavelengths of 488 and $530 \mathrm{~nm}$.

\section{Western blotting}

To reveal the mechanism of the apoptotic effect of TBMS1, Western blotting was done for apoptotic related proteins as previously described (Rasul et al., 2012b; Rasul et al., 2012c). Briefly, A375 cells were incubated with 20 and $40 \mu \mathrm{M}$ of TBMS1 for indicated time. Cells were trypsinized, collected in $1.5 \mathrm{~mL}$ centrifuge tube and washed with PBS. The cell pellets were resuspended in lysis buffer and were lysed on ice for $30 \mathrm{~min}$. After centrifugation for $15 \mathrm{~min}$, the supernatant fluids were collected and the protein content of the supernatant was measured by the NanoDrop 1000 spectrophotometer (Thermo scientific, USA). The protein lysates were separated by electrophoresis on $12 \%$ SDSpolyacrylamide gel and transferred to a PVDF membrane (Amersham Biosciences, Piscataway, NJ). The membranes were soaked in blocking buffer $(5 \%$ skimmed milk) for 2 hours. To probe for Bcl-2, Bax, and $\beta$-actin; membranes were incubated overnight at $4^{\circ} \mathrm{C}$ with relevant antibodies, followed by appropriate HRP conjugated secondary antibodies and ECL detection.

\section{Statistical analysis of data}

For the statistical analysis of data, comparisons between results from different groups were analyzed with SPSS for Window Version 15.0. Student's t-test was employed to determine the statistical significance of the difference between different experimental groups and control group. $p<0.05$ value was defined as statistically significant. All experiments were repeated at least three times. Data were presented as mean \pm standard deviation (SD).

\section{Results and Discussion}

The investigation was initiated with screening of natural compounds against A375 human melanoma cells. We found that TBMS1 exhibited cytotoxic effects on the growth of A375 cells. TBMS1 is a natural compound that belongs to a triterpenoid saponin family. We determined the effects of TBMS1 on the growth of A375 human melanoma cells by quantifying the viable cells using MTT assay. TBMS1 inhibited the growth of A375 human melanoma cells in a dose- dependent manner (Figure 2). Morphological changes were observed under phase contrast microscopy after treating cells with 20 and $40 \mu \mathrm{M}$ of TBMS1. There was a significant decrease in the number of A375 cells treated with TBMS1 as compared to the control group. Furthermore, the cells become round-shaped and poorly adhered to the cultured plates while the control group cells showed a typical polygonal and cobblestone monolayer appearance and remained firmly attached to cultured plates (data not shown). The results indicated that TBMS1 induced growth inhibition of A375 cells, in addition to other type of cancer cells previously reported including A549 lung cancer cells (Zhang et al., 2011), HeLa cervical cancer cells (Xu et al., 2009), and nasopharyngeal carcinoma CNE-2Z (Ma et al., 2008; Weng et al., 2003).

There are several mechanisms which control the cell cycle to ensure the correct cell division. It is well known that progression of cell cycle is maintained by different check points in normal cells and the transition from one cell cycle phase to another occurs in an orderly fashion. In cancerous cells, some basic modifications occurred in the genetic control of cell division, resulting in an uncontrolled cell proliferation. As the deregulation of cell cycle progression is the hallmark of cancer; thereby cell cycle regulation could be a potential and effective strategy for the treatment of cancer (Grana and Reddy, 1995; Vermeulen et al., 2003). Therefore, we analyzed effect of TBMS1 on cell cycle progression of A375 cells. It was found that TBMS1 arrested cell cycle at G2/M phase, which supported the previous reported results in HeLa cells (Xu et al., 2009). The percentage of accumulation of cells in the G2/M phase was increased from $9.4 \%$ in control group to $26.7 \%$, and $37.9 \%$ in the cells treated with 20 and $40 \mu \mathrm{M}$ of TBMS1 respectively for 24 hours (Figure 3). These findings revealed that G2/M phase cell cycle arrest was one of the mechanisms through which TBMS1 induces cytotoxicity in A375 cells. A number of recent studies have shown that by arresting the cell division at certain checkpoints in the cell cycle, several chemotherapeutic and chemopreventive agents have demonstrated potential antiproliferative effects (Mantena et al., 2006; Srivastava and Gupta, 2006; Vermeulen et al., 2003).

There are various modes of cell death such as apoptosis, autophagy and necrosis (Leist and Jaattela, 2001). 


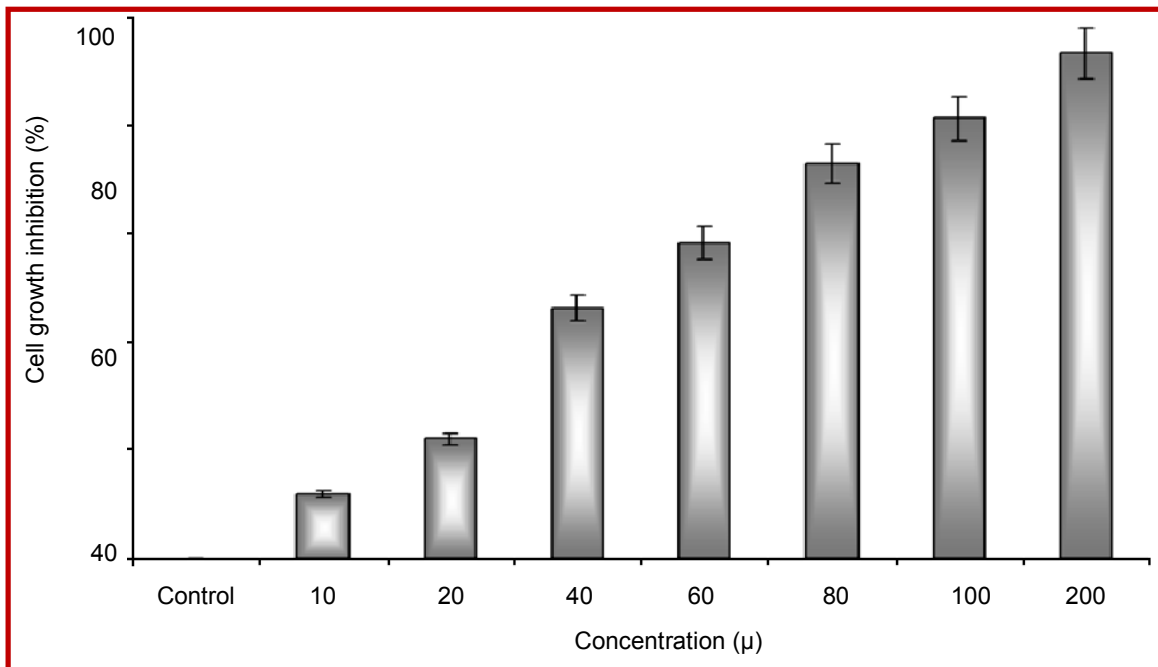

Figure 2: The effects of TBMS1 on viability of A375 cells. A375 cells were treated with various concentrations of TBMS1 for 24 hours. Cell death was determined by using MTT assay. Data shown are means \pm SD $(n=3)$

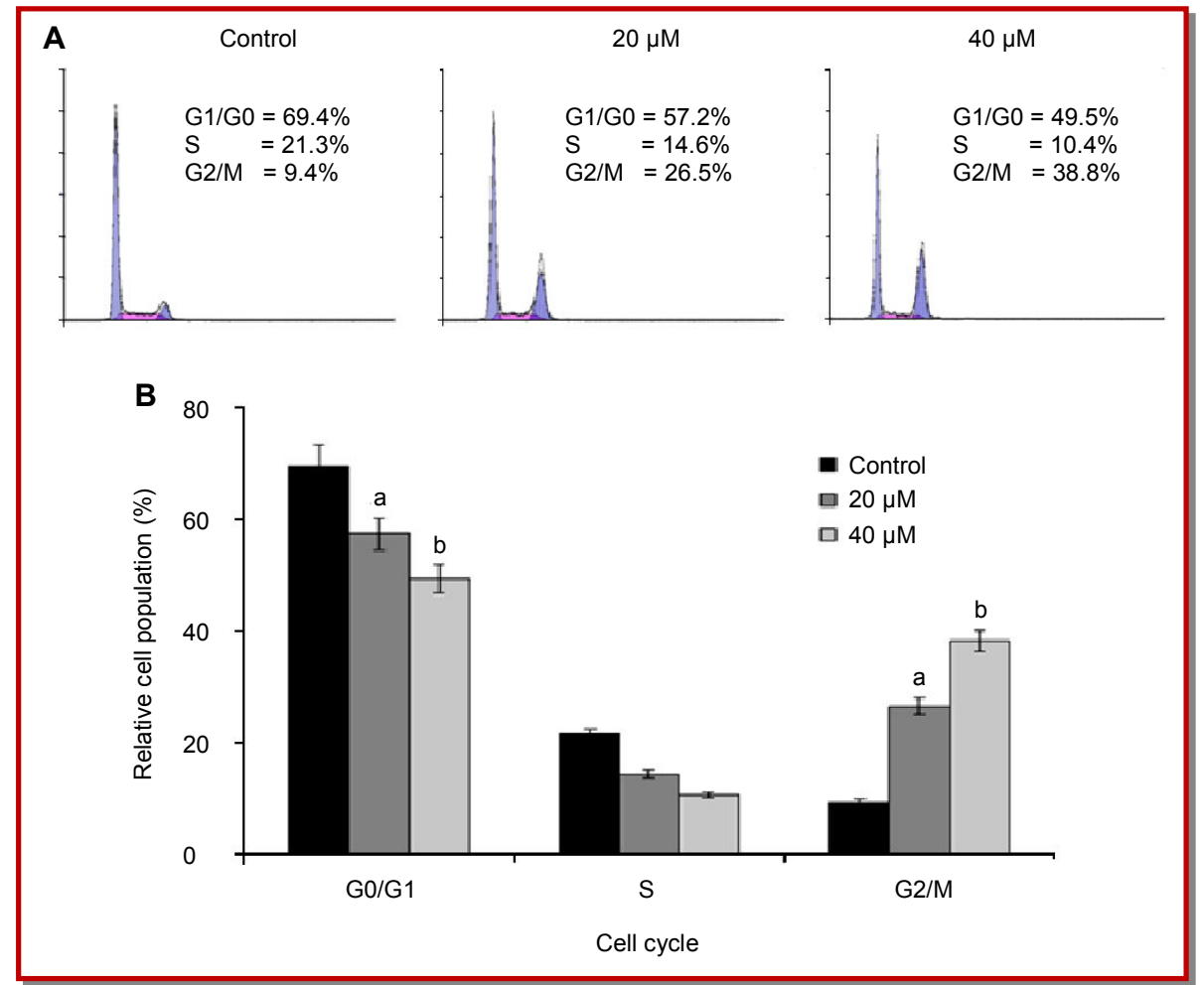

Figure 3: Effect of TBMS1 on the cell cycle distribution. A375 cells were treated with 0, 20, and $40 \mu \mathrm{M}$ of TBMS1 for 24 hours and then they were stained with PI for flow cytometric analysis. (A) Histograms show number of cells/channel ( $y$-axis) vs. DNA content $(x$-axis) and (B) The values indicate the percentage of cells in the indicated phases of cell cycle. The data shown are representative of three independent experiments with the similar results. a $p<0.05$ and ${ }^{b} p<0.01$ compared with the control

Apoptosis is most organized, well fashioned, and systematic mode of cell death, in which cells themselves play an active role in their own death (Elmore, 2007; Hengartner, 2000). The normal cellular signals for regulation of their growth are lost in the cancerous cells due to various mutations, preventing the cells from apoptosis and cell growth to uncontrolled status (Hanahan and Weinberg, 2000). The regulation of apoptosis is, therefore, most important in the treatment of cancer (Fulda, 2010; Lawen, 2003; Reed, 2002). The chemo- 


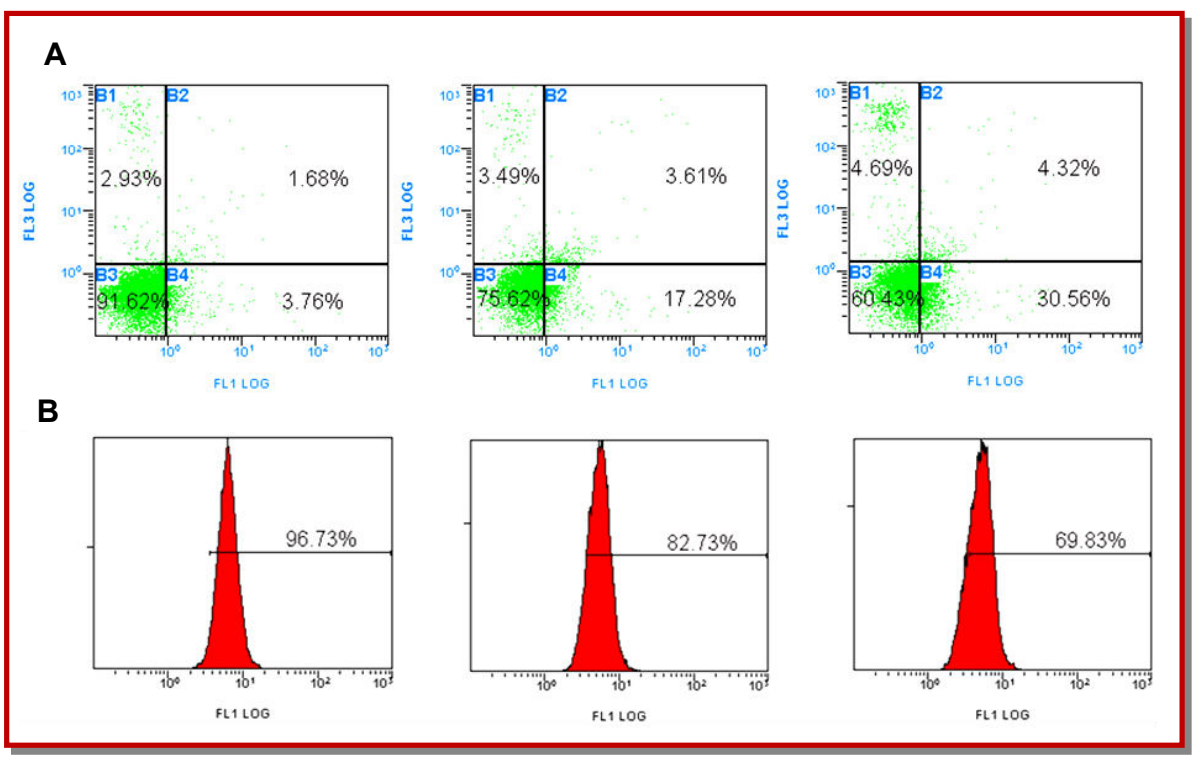

Figure 4: Effects of TBMS1 on apoptosis and mitochondrial transmembrane potential in A375 cells. (A) A375 cells were treated with 0,20 and $40 \mu \mathrm{M}$ of TBMS1 for 24 hours. Then they were stained with Annexin V-FITC/PI and were analysed in flow cy- tometry. Cell populations shown in the lower right (Annexin V+/PI-) represents early apoptotic cells, upper right (Annexin V+/ PI+) represents late apoptotic cells. (B) The values indicate the percentage of Rho-123 fluorescence in the A375 cells treated without (control) and with $(20$ and $40 \mu \mathrm{M})$ TBMS for 12 hours. The data shown are representative of three independent experiments with the similar results

preventive agents, which can treat the cancer effectively, have potential to restore the natural signaling apoptotic pathway (Reed, 1999). It is well known that various chemopreventive agents cause cell death through induction of apoptosis in different cancer cells (Srivastava and Gupta, 2006; Xu et al., 2009). We studied whether TBMS1 inhibits cell growth in A375 cells through the induction of apoptosis. TBMS1- induced apoptosis was determined by flow cytometric analysis.

For flow cytometric analysis, cells were seeded in the 12 well plates. After incubation of cells without (control) or with TBMS1 for 24 hours, cells were collected in centrifuged tubes and stained with annexin V-FITC and PI double staining as described in material and methods part. The results of flow cytometric analysis showed that rates of apoptosis were 20.9 and $34.9 \%$ in the cells treated with 20 and $40 \mu \mathrm{M}$ of TBMS1 respectively for 24 hours as compared to $4.6 \%$ in the control cells (Figure 4A). TBMS1-induced apoptosis in A375 cells was consistent with previously reported studies in lung and cervical cancer cells as well as in leukemic cells (Weng et al., 2003; Xu et al., 2009; Zhang et al., 2011).

Mitochondria play a fundamental role in the regulation of apoptotic cell death and consist of various proapoptotic proteins and cytochrome $c$. As highlighted earlier, apoptosis involves a dysfunction of mitochondrial membrane integrity, which leads to cell death (Jeong and Seol, 2008). Previously it has been documented that disintegration of the mitochondrial membrane potential and the redistribution of cytochrome c. are crucial actions in the apoptotic cascade (Kluck et al., 1997; Wang, 2001). An abrupt decrease of $\Delta \Psi \mathrm{m}$ was found in some anti-cancer compoundsinduced apoptosis in cancer cells (Chen et al., 2007). To probe the effect of TBMS1 on the $\Delta \Psi \mathrm{m}, \mathrm{A} 375$ cells were stained with the Rho-123 to measure the mitochondrial transmembrane potential. The loss of mitochondrial membrane potential was considered directly proportional to the decrease of Rhodamine- 123 fluorescence in treated cells as compared to the normal cells. The fluorescence intensity of Rhodamine-123 was observed by flow cytometry which was 82.7 and $68.1 \%$ after treating cells with 20 and $40 \mu \mathrm{M}$ of TBMS1 respectively for 12 hours in comparison to $96.7 \%$ in the untreated cells (Figure 4B). The results indicated depletion of mitochondrial transmembrane potential by TBMS1 in a dose-dependent manner in A375 cells.

Cytochrome $c$ plays central role in mitochondrial mediated apoptosis. Upon the attenuation of mitochondrial transmembrane potential, Cytochrome c releases from the mitochondria into the cytosol (Kluck et al., 1997). Once released into the cytosol, cytochrome $c$ binds to form an "apoptosome" of Apaf-1, cytochrome $c$, and caspase-9, which subsequently cleaves the effecter caspase-3 (Ricci and Zong, 2006).

The release of cytochrome $\mathrm{c}$ from the mitochondria into cytosol is coupled with the ratio of $\mathrm{Bax} / \mathrm{Bcl}-2$ proteins (Kluck et al., 1997). Bcl-2 family proteins are mostly involved in the mitochondrial apoptotic pathway 


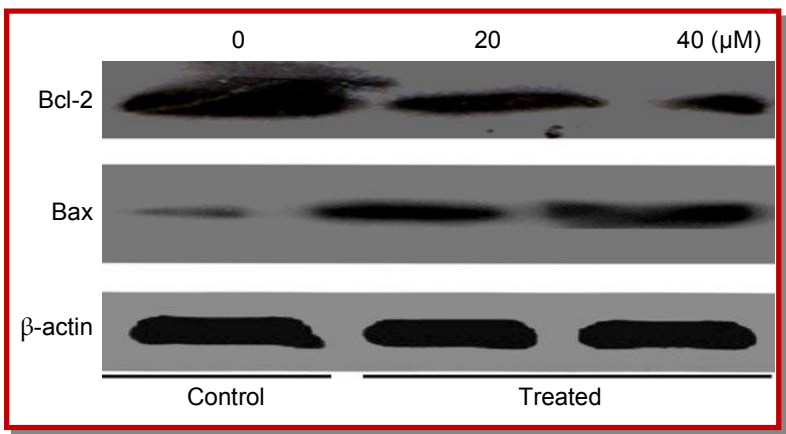

Figure 5: The effects of TBMS1 on expression levels of apoptosis related proteins. Expression levels of Bcl-2 and Bax in A375 cells treated without (control) and with $(20$ and $40 \mu \mathrm{M})$ TBMS for 24 hours were monitored by Western blot assay. $\beta$-actin was used as loading control. Western blots are representative of three independent experiments

(Reed, 1998). Furthermore, Bcl-2 family plays a central role in activation of Caspases (Burlacu, 2003). Bcl-2 and Bax work in an antagonistic manner, Bcl-2 is antiapoptotic while Bax is proapoptotic member of Bcl2 family and Bcl-2 forms heterodimers with Bax. Bax inserts into outer membrane of mitochondria under stress conditions, as a results transmembrane permeability become higher which facilitate the release of cytochrome $C$ due to formation of pores on the mitochondrial outer membrane (Adams and Cory, 2007; Danial, 2007). The balance between these two groups is critical in the cell decision to undergo apoptosis or not (Mallat and Tedgui, 2000). We performed western blotting to examine the effect of TBMS1 on the expression of Bcl-2 and Bax. TBMS1 was observed to be involved in the up-regulation of Bax and down regulation of Bcl-2 in dose-dependent manner (Figure $5)$. These results are similar with previously reported studies in Hela cells (Xu et al., 2009).

\section{Conclusion}

TBMS1 induced apoptosis of A375 human melanoma cells accompanied by a G2/M phase cell cycle arrest. TBMS1 induced loss of mitochondrial membrane potential via down-regulation of $\mathrm{Bcl}-2$ and upregulation of Bax, which facilitate the release of cytochrome $c$ into cytosol due to the depolarization of mitochondrial membranes. These in vitro results suggested that TBMS1 should be further examined for in vivo activity and molecular mechanism in human melanoma.

\section{Acknowledgement}

This study was supported by Ministry of Science and Technology (No. 2010DFA31430), NCET-10-0316; the Fundamental Research Funds for the Central University
(10JCXK004), and Changchun Science \& Technology Department (No. 2011114).

\section{References}

Adams JM, Cory S. The Bcl-2 apoptotic switch in cancer development and therapy. Oncogene 2007; 26: 1324-37.

Amin AR, Kucuk O, Khuri FR, Shin DM. Perspectives for cancer prevention with natural compounds. J Clin Oncol. 2009; 27: $2712-25$.

Burlacu A. Regulation of apoptosis by Bcl-2 family proteins. J Cell Mol Med. 2003; 7: 249-57.

Chen CY, Liu TZ, Liu YW, Tseng WC, Liu RH, Lu FJ, Lin YS, Kuo $\mathrm{SH}$, Chen $\mathrm{CH}$. 6-shogaol (alkanone from ginger) induces apoptotic cell death of human hepatoma p53 mutant Mahlavu subline via an oxidative stress-mediated caspasedependent mechanism. J Agric Food Chem. 2007; 55: 948-54.

Cragg GM, Newman DJ. Plants as a source of anti-cancer agents. J Ethnopharmacol. 2005; 100: 72-79.

Danial NN. BCL-2 family proteins: Critical checkpoints of apoptotic cell death. Clin Cancer Res. 2007; 13: 7254-63.

Elmore S. Apoptosis: A review of programmed cell death. Toxicol Pathol. 2007; 35: 495-516.

Fulda S. Evasion of apoptosis as a cellular stress response in cancer. Int J Cell Biol. 2010; 2010: 370835.

Grana X, Reddy EP. Cell cycle control in mammalian cells: Role of cyclins, cyclin dependent kinases (CDKs), growth suppressor genes and cyclin-dependent kinase inhibitors (CKIs). Oncogene 1995; 11: 211-19.

Gray-Schopfer V, Wellbrock C, Marais R. Melanoma biology and new targeted therapy. Nature 2007; 445: 851-57.

Hanahan D, Weinberg RA. The hallmarks of cancer. Cell 2000; 100: 57-70.

Hengartner MO. The biochemistry of apoptosis. Nature 2000; 407: 770-76.

Hofmann MA, Sterry W, Trefzer U. Complex combination biochemotherapy regimen in advanced metastatic melanoma in a non-intensive care unit: toxicity or benefit? Jpn J Clin Oncol. 2007; 37: 224-29.

Ives NJ, Stowe RL, Lorigan P, Wheatley K. Chemotherapy compared with biochemotherapy for the treatment of metastatic melanoma: A meta-analysis of 18 trials involving 2,621 patients. J Clin Oncol. 2007; 25: 5426-34.

Jeong SY, Seol DW. The role of mitochondria in apoptosis. BMB Rep. 2008; 41: 11-22.

Kluck RM, Bossy-Wetzel E, Green DR, Newmeyer DD. The release of cytochrome $\mathrm{c}$ from mitochondria: A primary site for Bcl-2 regulation of apoptosis. Science 1997; 275: 1132-36.

Lawen A. Apoptosis-an introduction. Bioessays 2003; 25: 88896.

Legha SS, Ring S, Bedikian A, Plager C, Eton O, Buzaid AC, Papadopoulos N. Treatment of metastatic melanoma with combined chemotherapy containing cisplatin, vinblastine 
and dacarbazine (CVD) and biotherapy using interleukin-2 and interferon-alpha. Ann Oncol. 1996; 7: 827-35.

Leist M, Jaattela M. Four deaths and a funeral: From caspases to alternative mechanisms. Nat Rev Mol Cell Biol. 2001; 2: 589-98.

Ma R, Song G, You W, Yu L, Su W, Liao M, Zhang Y, Huang L, Zhang X, Yu T. Anti-microtubule activity of tubeimoside I and its colchicine binding site of tubulin. Cancer Chemother Pharmacol. 2008; 62: 559-68.

Magadula JJ, Erasto P. Bioactive natural products derived from the East African flora. Nat Prod Rep. 2009; 26: 1535-54.

Mallat Z, Tedgui A. Apoptosis in the vasculature: Mechanisms and functional importance. Br J Pharmacol. 2000; 130: 947-62.

Mantena SK, Sharma SD, Katiyar SK. Berberine, a natural product, induces G1-phase cell cycle arrest and caspase-3dependent apoptosis in human prostate carcinoma cells. Mol Cancer Ther. 2006; 5: 296-308.

Rasul A, Khan M, Yu B, Ma T, Yang H. Xanthoxyletin, a coumarin induces $S$ phase arrest and apoptosis in human gastric adenocarcinoma SGC-7901 cells. Asian Pac J Cancer Prev. 2011a; 12: 1219-23.

Rasul A, Parveen S, Ma T. Costunolide: A novel anti-cancer sesquiterpene lactone. Bangladesh J Pharmacol. 2012a; 7: 613.

Rasul A, Yu B, Khan M, Zhang K, Iqbal F, Ma T, Yang H. Magnolol, a natural compound, induces apoptosis of SGC7901 human gastric adenocarcinoma cells via the mitochondrial and PI3K/Akt signaling pathways. Int J Oncol. 2012b; 40: 1153-61.

Rasul A, Yu B, Yang LF, Ali M, Khan M, Ma T, Yang H. Induction of mitochondria-mediated apoptosis in human gastric adenocarcinoma SGC-7901 cells by kuraridin and Nor-kurarinone isolated from Sophora flavescens. Asian Pac J Cancer Prev. 2011b; 12: 2499-2504.

Rasul A, Yu B, Zhong L, Khan M, Yang H, Ma T. Cytotoxic effect of evodiamine in SGC-7901 human gastric adenocarcinoma cells via simultaneous induction of apoptosis and autophagy. Oncol Rep. 2012c; 27: 1481-87.

Reed JC. Bcl-2 family proteins. Oncogene 1998; 17: 3225-36.

Reed JC. Dysregulation of apoptosis in cancer. J Clin Oncol.
1999; 17: 2941-53.

Reed JC. Apoptosis-based therapies. Nat Rev Drug Discov. 2002; 1: 111-21.

Ricci MS, Zong WX. Chemotherapeutic approaches for targeting cell death pathways. Oncologist 2006; 11: 342-57.

Srivastava JK, Gupta S. Tocotrienol-rich fraction of palm oil induces cell cycle arrest and apoptosis selectively in human prostate cancer cells. Biochem Biophys Res Commun. 2006; 346: 447-53.

Vermeulen K, Van Bockstaele DR, Berneman ZN. The cell cycle: A review of regulation, deregulation and therapeutic targets in cancer. Cell Prolif. 2003; 36: 131-49.

Wang $X$. The expanding role of mitochondria in apoptosis. Genes Dev. 2001; 15: 2922-33.

Weng XY, Ma RD, Yu LJ. [Apoptosis of human nasopharyngeal carcinoma CNE-2Z cells induced by tubeimoside I]. Ai Zheng. 2003; 22: 806-11.

Woldemichael GM, Wink M. Identification and biological activities of triterpenoid saponins from Chenopodium quinoa. J Agric Food Chem. 2001; 49: 2327-32.

$\mathrm{Xu} \mathrm{Y,} \mathrm{Chiu} \mathrm{JF,} \mathrm{He} \mathrm{QY,} \mathrm{Chen} \mathrm{F.} \mathrm{Tubeimoside-1} \mathrm{exerts}$ cytotoxicity in HeLa cells through mitochondrial dysfunction and endoplasmic reticulum stress pathways. J Proteome Res. 2009; 8: 1585-93.

$\mathrm{Yu}$ L, Ma R, Yu T. Induction of morphological and functional differentiation of human promyelocytic leukemia cells (HL60) by Tubeimoside 1. Planta Med. 1996; 62: 119-21.

Yu LJ, Ma RD, Jiang SB. Effects of tubeimoside-1 on HIV core protein 24 and cytopathogenesis in vitro. Zhongguo Yao Li Xue Bao. 1994; 15: 103-06.

Yu LJ, Ma RD, Wang YQ, Nishino H, Takayasu J, He WZ, Chang M, Zhen J, Liu WS, Fan SX. Potent anti-tumorigenic effect of tubeimoside 1 isolated from the bulb of Bolbostemma paniculatum (Maxim) Franquet. Int J Cancer. 1992; 50: 635-38.

Zhang Y, Xu X, He P. Tubeimoside-1 inhibits proliferation and induces apoptosis by increasing the Bax to Bcl-2 ratio and decreasing COX-2 expression in lung cancer A549 cells. Mol Med Report. 2011; 4: 25-29.

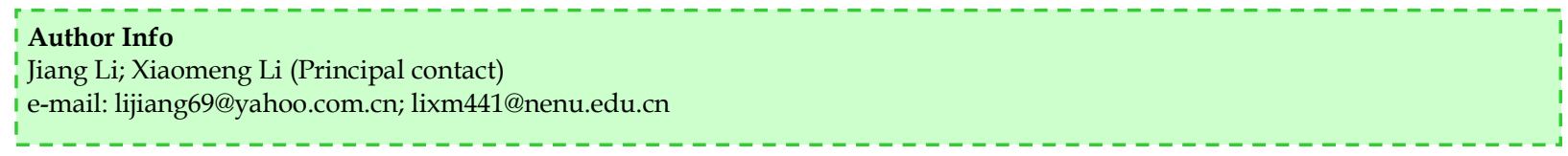

